\title{
HIVIAIDS-related lymphoma: perspective from a regional cancer center in India
}

\author{
A.H. Rudresha ${ }^{1}$, Pravin Ashok Khandare ${ }^{1}$, D. Lokanatha ${ }^{1}$, Abraham Jacob Linu ${ }^{1}$, M.C. Suresh Babu ${ }^{1}$, \\ K.N. Lokesh ${ }^{1}$, L.K. Rajeev ${ }^{1}$, Carol Saldanha Smitha ${ }^{1}$, Vaibhav Baburao Amale ${ }^{1}$, C.S. Premalata ${ }^{2}$, \\ Mulchandani Nikita ${ }^{2}$ \\ Departments of ${ }^{1}$ Medical Oncology, ${ }^{2}$ Pathology, Kidwai Cancer Institute, Bengaluru, India
}

p-ISSN 2287-979X / e-ISSN 2288-0011 https://doi.org/10.5045/br.2019.54.3.181 Blood Res 2019;54:181-188.

Received on March 25, 2019 Revised on April 18, 2019 Accepted on May 29, 2019

\section{Correspondence to}

Pravin Ashok Khandare, M.D. Department of Medical Oncology, Kidwai Cancer Institute, Dr M H, Marigowda Rd, Bengaluru, Karnataka 56002, India E-mail: pravin.gmc@gmail.com

(C) 2019 Korean Society of Hematology

\section{Background}

India has the third largest population of people living with HIV/AIDS (PLHA). Lymphoma is the second most common malignancy among PLHA. However, data are lacking regarding HIV/AIDS-related lymphoma (ARL) in India. This study evaluated the epidemiology and clinical outcomes of ARL from a regional cancer center in India.

\section{Methods}

This retrospective analysis included cases of ARL between March 2011 and September 2017. Data were obtained from patient record files for the assessment of epidemiology and clinical outcomes. Statistical analysis was performed using GraphPad Prism 6. Comparisons of subtype-specific survivals were performed using log-rank tests.

Results

Of 1,226 lymphoma cases, 80 (6.5\%) were ARL. Details were available for 70 patients. The median age at diagnosis was 40.5 (9-74) years with a male:female ratio of 2:1. AIDS-defining lymphomas (ADL) constituted $78.6 \%$ of cases, while $21.4 \%$ had non-AIDS defining lymphoma (NADL). The mean CD4 counts were 193.15 \pm 92.85 and $301.93 \pm 107.95$ cells $/ \mu \mathrm{L}$, respectively (t-test; $P=0.0002$ ). Extranodal involvement was present in $55.7 \%$, B symptoms were reported in $60 \%$, and lactate dehydrogenase (LDH) was elevated in $64.3 \%$ of patients. The median overall survival times were 6 months for plasmablastic lymphoma (PBL), 23 months for diffuse large B-cell lymphoma (DLBCL), and was not reached for Hodgkin's lymphoma (log-rank test; $P=0.0011$ ). Other histological subtype cases were too few to draw meaningful survival outcomes.

\section{Conclusion}

ARL is a heterogeneous disease. Histologic subtype is a major determinant of the clinical outcome. ADL has significantly lower CD4 counts than those of NADL. There is an urgent and unmet need for uniform management guidelines for improving outcomes in this under-represented patient population.

Key Words HIV, AIDS, Lymphoma, AIDS-related lymphoma

\section{INTRODUCTION}

India is the second most populated country in the world. According to the 2018 Joint United Nations Programme on HIV/AIDS (UNAIDS) report, with an HIV/AIDS prevalence rate of $0.3 \%$, India ranks at 80 th position in the world, and 2.1 million people have HIV/AIDS in India [1]. It is home to the third largest population of people living with
HIV/AIDS (PLHA) because of its large population size. The HIV epidemic in India is heterogeneous and concentrated in specific regions and high-risk populations. This epidemic is slowing, with a $33.5 \%$ decrease in the rate of new infections as per the National AIDS Control Organization (NACO) 2016-17 annual report compared to 2007 data [2]. Even with these improving statistics, HIV/AIDS is a public health problem in India, consuming a sizeable expenditure of the total health budget, which itself is meager, with only $1.15 \%$ of 
the gross domestic product (GDP) spent on public health [3].

The exact burden of HIV/AIDS-associated cancers in India is not known. Kaposi's sarcoma is the most frequent AIDS-defining cancer in the Western world, while non-Hodgkin's lymphoma (NHL) is most commonly reported in Indian literature [4-6]. We undertook the current study to evaluate the epidemiology and outcomes of HIV/AIDS-related lymphoma (ARL) in a tertiary cancer center in India.

\section{MATERIALS AND METHODS}

This was a retrospective analysis of patients who were diagnosed with lymphoma and were PLHA or who were diagnosed with HIV during the initial evaluation for their lymphoma between March 2011 and September 2017 (6.5 yr) at Kidwai Cancer Institute, a tertiary cancer center in India.

Written informed consent was obtained from all patients before the clinical examination, any procedure, and the start of treatment. Data including the demographic profile, clinical history and physical examination, staging, and treatment outcomes were collected from patient record files obtained from the medical records department. For known PLHA patients, the most recent CD4 count was recorded from patient treatment booklets provided by NACO and fresh blood samples were sent for newly diagnosed HIV patients for the estimation of CD4 counts. The laboratory investigations included complete blood count (CBC), liver function test (LFT), kidney function test (KFT), and serological tests for $\mathrm{HIV}$ and hepatitis B and C. The staging was done as per Cotswold's modification of the Ann Arbor staging system. Contrast-enhanced computed tomography (CECT) of the neck, thorax, abdomen, and pelvis was used at baseline and for response assessment at regular intervals and at the completion of treatment, for most patients. ${ }^{18}$ Fluoro-2-deoxy-d-glucose positron emission tomography-computed to- mography $\left({ }^{18}\right.$ FDG PET-CT) was used in a small minority of cases because of logistic and financial constraints. Other investigations including cerebrospinal fluid (CSF) examination, unilateral bone marrow aspiration and biopsy, and magnetic resonance imaging (MRI) of the brain were performed as necessary. Histopathological examination of tissue samples, either of lymph node or extranodal sites, was done by an expert pathologist for diagnosis. Immunohistochemical (IHC) markers were used for confirmation and further characterization based on morphology. Treatment was based on final histopathological diagnosis, using standard chemotherapy regimens as per guidelines.

Patients in this study were divided into AIDS-defining lymphomas (ADL) and non-AIDS defining lymphomas (NADL) groups. ADL included diffuse large B-cell lymphoma (DLBCL), plasmablastic lymphoma (PBL), high-grade B-cell lymphoma-not otherwise specified (NOS), Burkitt's lymphoma (BL), primary effusion lymphoma (PEL), and primary central nervous system lymphoma (PCNSL). NADL included Hodgkin's lymphoma (HL), follicular lymphoma (FL), and peripheral T-cell lymphoma-NOS (PTCL).

The outcomes were measured in terms of progression-free survival (PFS) and overall survival (OS) using the KaplanMeier method. PFS was calculated from the date of diagnosis until clinical or radiological disease progression, death from any cause, or last follow-up, as appropriate. OS was measured from the date of diagnosis until the date of death from any cause. If no event occurred, data were censored at the time of the last follow-up visit. Adverse events were graded as per National Cancer Institute Common Terminology Criteria for Adverse Events (NCI CTCAE) version 4.03. Statistical analysis was performed using GraphPad Prism (version 6, GraphPad Software, San Diego, CA, USA).

\section{RESULTS}

A total of 1,226 lymphoma cases were registered at our

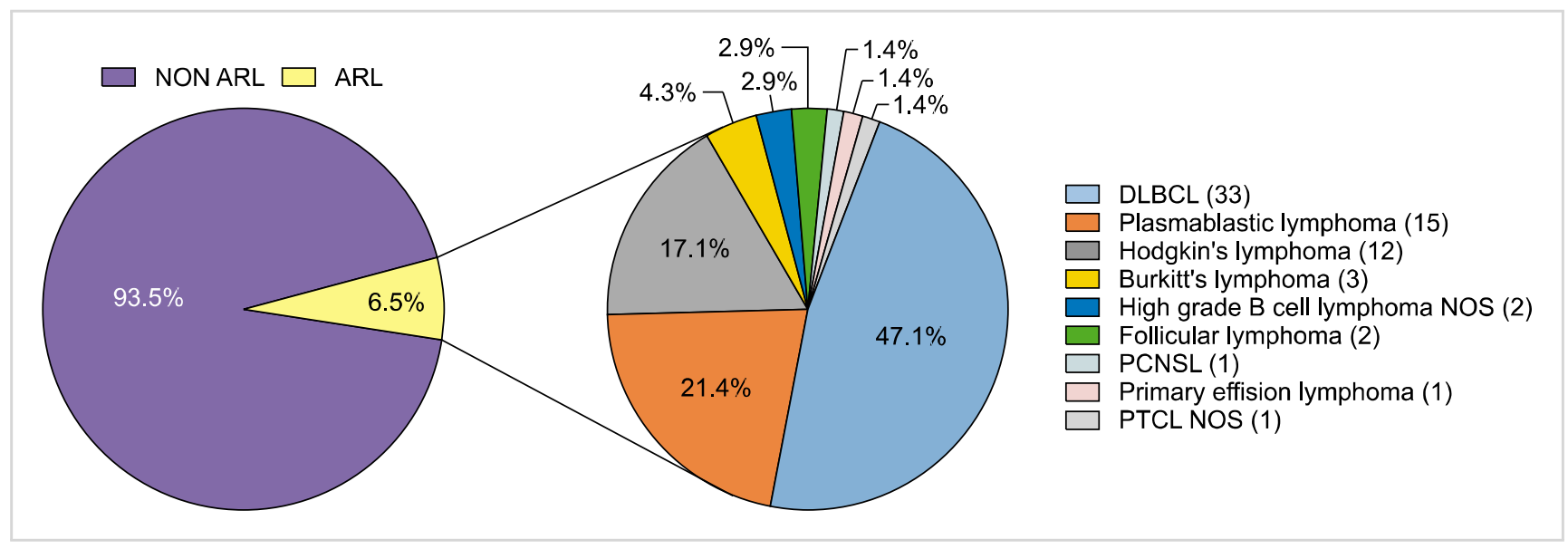

Fig. 1. Distribution of HIV/AIDS-related lymphoma.

Abbreviations: DLBCL, diffuse large B-cell lymphoma; NOS, not otherwise specified; PCNSL, primary central nervous system lymphoma; PTCL NOS, peripheral T-cell lymphoma-NOS. 
center during the 6.5-year period between March 2010 to August 2017. Eighty (6.5\%) cases were clinically diagnosed as ARL. Details were available for 72 of these cases; two patients had a final histopathological diagnosis of atypical lymphoid hyperplasia, while 70 were diagnosed with lymphoma. These 70 patients were included in the final analysis.

The median age at diagnosis for the entire cohort was 40.5 (9-74) years, with a male to female ratio of 2:1. Fifty-five (78.6\%) patients had ADL while 15 (21.4\%) had NADL. The most common histologies were DLBCL (47.1\%), PBL (21.4\%), and HL (17.1\%). Survival analysis was performed for these three major histologies. Fig. 1 shows the distribution of ARL. The number of patients with other histologies was too few to draw meaningful conclusions regarding clinical outcomes. The baseline characteristics of the patients are shown in Table 1.

Of the 70 patients, lymphoma was presenting complaint of underlying HIV infection in 30 (42.9\%), while 40 (57.1\%) were known PLHA and on combination antiretroviral therapy (ART). The most common ART regimen was the fixed drug combination of tenofovir, lamivudine, and efavirenz and all patients were taking treatment with registered ART centers under NACO. All newly diagnosed HIV patients were referred from our institute to a nearby registered ART center for baseline evaluation of HIV and to start ART. Further treatment for HIV/AIDS was done from registered ART centers nearest to their place of residence. Five patients had concurrent active hepatitis $\mathrm{B}$ and one patient had a hepatitis $\mathrm{C}$ infection. History of pulmonary or extrapulmonary tuberculosis (TB) was present in 22 (31.42\%) patients, while four $(5.7 \%)$ patients had active $\mathrm{TB}$ and were on anti-TB treatment. Patients on anti-TB treatment were taking ART with a fixed drug combination of zidovudine, lamivudine, and efavirenz, as per NACO guidelines. Patients continued ART throughout and after completion of lymphoma treatment.

Among the entire cohort, extranodal involvement was present in $55.7 \%$ of patients, with bone marrow the most common site, followed by the gastrointestinal tract. The other common extranodal sites were bone, central nervous system (CNS), and oral cavity. Lactate dehydrogenase (LDH) levels were elevated in $64.3 \%$ of patients. B symptoms were reported in $60 \%$ of patients. The mean CD4 counts (cells $/ \mu \mathrm{L}$ ) were $193.15 \pm 92.85$ and $301.93 \pm 107.95$ in ADL and NADL, respectively, with a significant difference (t-test; $P=0.0002$ ).

DLBCL patients were treated with anthracycline-based regimens which included CHOP (cyclophosphamide, hydroxydaunorubicin, vincristine, and prednisone) and DA-EPOCH (dose-adjusted etoposide, prednisone, vincristine, cyclophosphamide, and hydroxydaunorubicin). Only six $(18.2 \%)$ patients received rituximab-based treatment. PBL patients were treated with either CHOP or DA-EPOCH. Of 12 patients with HL, 11 received ABVD (adriamycin, bleomycin, vinblastine, and dacarbazine), while one received COPP (cyclophosphamide, vincristine, procarbazine, and

Table 1. Baseline patient characteristics.

\begin{tabular}{|c|c|c|c|c|}
\hline Characteristic & DLBCL $(\mathrm{N}=33)$ & $\mathrm{PBL}(\mathrm{N}=15)$ & $\mathrm{HL}(\mathrm{N}=12)$ & Total $(\mathrm{N}=70)$ \\
\hline Age, median (range) & $43 \mathrm{yr}(9-67)$ & 40 yr (12-64) & 40 yr (25-60) & 40.5 yr (9-74) \\
\hline $\mathrm{M}: \mathrm{F}$ & $1.2: 1$ & $6.5: 1$ & $1.4: 1$ & $2: 1$ \\
\hline \multicolumn{5}{|l|}{ ECOG PS } \\
\hline 0 & $0(0 \%)$ & $0(0 \%)$ & $0(0 \%)$ & $0(0 \%)$ \\
\hline 1 & $18(54.5 \%)$ & $6(40 \%)$ & $8(66.6 \%)$ & $37(52.8 \%)$ \\
\hline 2 & $10(30.3 \%)$ & $7(46.6 \%)$ & $4(33.3 \%)$ & $26(37.1 \%)$ \\
\hline 3 & $4(12.1 \%)$ & $2(13.3 \%)$ & $0(0 \%)$ & 7 (10\%) \\
\hline \multicolumn{5}{|l|}{ Stage } \\
\hline 1 & $5(15.1 \%)$ & $3(20 \%)$ & $1(8.3 \%)$ & $11(15.7 \%)$ \\
\hline II & $7(21.2 \%)$ & $8(53.3 \%)$ & $4(33.3 \%)$ & $17(24.3 \%)$ \\
\hline III & $8(24.2 \%)$ & $1(6.6 \%)$ & $5(41.6 \%)$ & $15(21.4 \%)$ \\
\hline IV & $10(30.3)$ & $2(13.3 \%)$ & $2(16.6 \%)$ & $21(30 \%)$ \\
\hline NA & $3(9 \%)$ & $1(6.6 \%)$ & $0(0 \%)$ & $6(8.6 \%)$ \\
\hline B symptoms & $21(63.3 \%)$ & $6(40 \%)$ & $9(75 \%)$ & $42(60 \%)$ \\
\hline LDH (elevated) & $20(60.6 \%)$ & $13(86.6 \%)$ & $4(33.3 \%)$ & $45(64.2 \%)$ \\
\hline IPI & & & IPS & NA \\
\hline Low & $5(15.1 \%)$ & $2(13.3 \%)$ & & \\
\hline Intermediate & $12(36.3 \%)$ & $7(46.6 \%)$ & $<3=8(66.6 \%)$ & \\
\hline High & $13(39.4 \%)$ & $5(33.3 \%)$ & $\geq 3=4(33.3 \%)$ & \\
\hline NA & $3(9 \%)$ & $1(6.6 \%)$ & & \\
\hline Mean CD4 count & $244 \mathrm{cell} / \mu \mathrm{L}$ & $129 \mathrm{cell} / \mu \mathrm{L}$ & $294 \mathrm{cell} / \mu \mathrm{L}$ & $216 \mathrm{cell} / \mu \mathrm{L}$ \\
\hline Extranodal involvement & $19(57.6 \%)$ & $11(73.3 \%)$ & $3(25 \%)$ & $39(55.7 \%)$ \\
\hline
\end{tabular}

Abbreviations: DLBCL, diffuse large B-cell lymphoma; ECOG PS, Eastern Cooperative Oncology Group Performance Status Scale; HL, Hodgkin's lymphoma; IPI, International Prognostic Index; IPS, International Prognostic Score; LDH, lactate dehydrogenase; NA, not applicable; $\mathrm{PBL}$, plasmablastic lymphoma. 
prednisone). CNS prophylaxis with $12 \mathrm{mg}$ of intrathecal (IT) methotrexate was administered to all DLBCL patients with each chemotherapy cycle and three patients with positive CSF cytology received triple IT with methotrexate $(12 \mathrm{mg})$, hydrocortisone $(50 \mathrm{mg})$, and etoposide $(70 \mathrm{mg})$, twice weekly until CSF clearance, weekly thereafter for four doses, and then once each cycle. The treatments and outcomes are depicted in Table 2.

Eleven (17.2\%) patients died during induction treatment, six and five in the DLBCL and PBL subgroups, respectively. At least one episode of grade $3 / 4$ hematological toxicity was experienced by $82 \%$ of patients. Febrile neutropenia was documented in $60 \%$ of patients. At the time of analysis, the median PFS and OS were 19 and 23 months, respectively, for the entire cohort. The median PFS was five and 19 months and the median OS was six and 23 months, respectively, for PBL and DLBCL. The median PFS and OS were not reached for HL. Histologic subtype specific-survival differed significantly for PFS (log-rank test; $P=0.0012$ ) and OS (log-rank test; $P=0.0011$ ). Fig. 2 and 3 show the Kaplan-Meier survival curves for the entire cohort and specific-histology subtypes, respectively. Comparison of the outcomes of 33 patients with DLBCL was done based on rituximab use $(\mathrm{N}=6)$ or no use $(\mathrm{N}=27)$. Though numerically higher, there was no significant difference in PFS and OS based on rituximab use (log-rank test; $P=0.075$ for PFS, $P=0.089$ for OS). Only three patients with DLBCL and one patient with PBL progressed or relapsed after first-line treatment received second-line treatment, while the majority who progressed or relapsed received best supportive care or defaulted and were lost to follow-up.

Table 2. Treatments and outcomes.

\begin{tabular}{|c|c|c|c|c|c|c|}
\hline \multirow{2}{*}{$\begin{array}{c}\text { Subtype } \\
\text { Treatment received }\end{array}$} & \multicolumn{2}{|c|}{ DLBCL, N (\%) } & \multicolumn{2}{|c|}{ PBL, N (\%) } & \multicolumn{2}{|c|}{$\mathrm{HL}, \mathrm{N}(\%)$} \\
\hline & $\mathrm{CHOP}$ & $22(66.6 \%)$ & $\mathrm{CHOP}$ & $10(66.6 \%)$ & ABVD & $11(91.6 \%)$ \\
\hline & $\mathrm{R}$ CHOP & $5(15.5 \%)$ & DA-EPOCH & $5(33.3 \%)$ & COPP & $1(8.3 \%)$ \\
\hline & DA-EPOCH & $5(15.5 \%)$ & & & & \\
\hline & R DA-EPOCH & $1(3 \%)$ & & & & \\
\hline \multirow[t]{6}{*}{ Treatment outcomes } & CR & 18 (54.5\%) & CR & $1(6.6 \%)$ & CR & $12(100 \%)$ \\
\hline & PR & $2(6 \%)$ & PR & $1(6.6 \%)$ & PR & 0 \\
\hline & SD & $1(3 \%)$ & SD & $4(26.6 \%)$ & SD & 0 \\
\hline & PD & $4(12.1 \%)$ & PD & $4(26.6 \%)$ & PD & 0 \\
\hline & Unknown 2 (6\%) & $4(12.1 \%)$ & Unknown & $1(6.6 \%)$ & Unknown & 0 \\
\hline & Induction death & $6(18.1 \%)$ & Induction death & $5(33.3 \%)$ & Induction death & 0 \\
\hline Median PFS & \multicolumn{2}{|c|}{$19 \mathrm{mo}$} & \multicolumn{2}{|c|}{$5 \mathrm{mo}$} & \multicolumn{2}{|c|}{ NR } \\
\hline Median OS & \multicolumn{2}{|c|}{$23 \mathrm{mo}$} & \multicolumn{2}{|c|}{$6 \mathrm{mo}$} & \multicolumn{2}{|c|}{ NR } \\
\hline
\end{tabular}

Abbreviations: ABVD, adriamycin, bleomycin, vinblastine, and dacarbazine; $\mathrm{CHOP}$, cyclophosphamide, hydroxydaunorubicin, vincristine, and prednisone; CR, complete response; DA-EPOCH, dose-adjusted etoposide, prednisone, vincristine, cyclophosphamide, and hydroxydaunorubicin; DLBCL, diffuse large B-cell lymphoma; NR, not reached; OS, overall survival; PBL, plasmablastic lymphoma; PD, progressive disease; PFS, progression-free survival; PR, partial response; SD, stable disease.
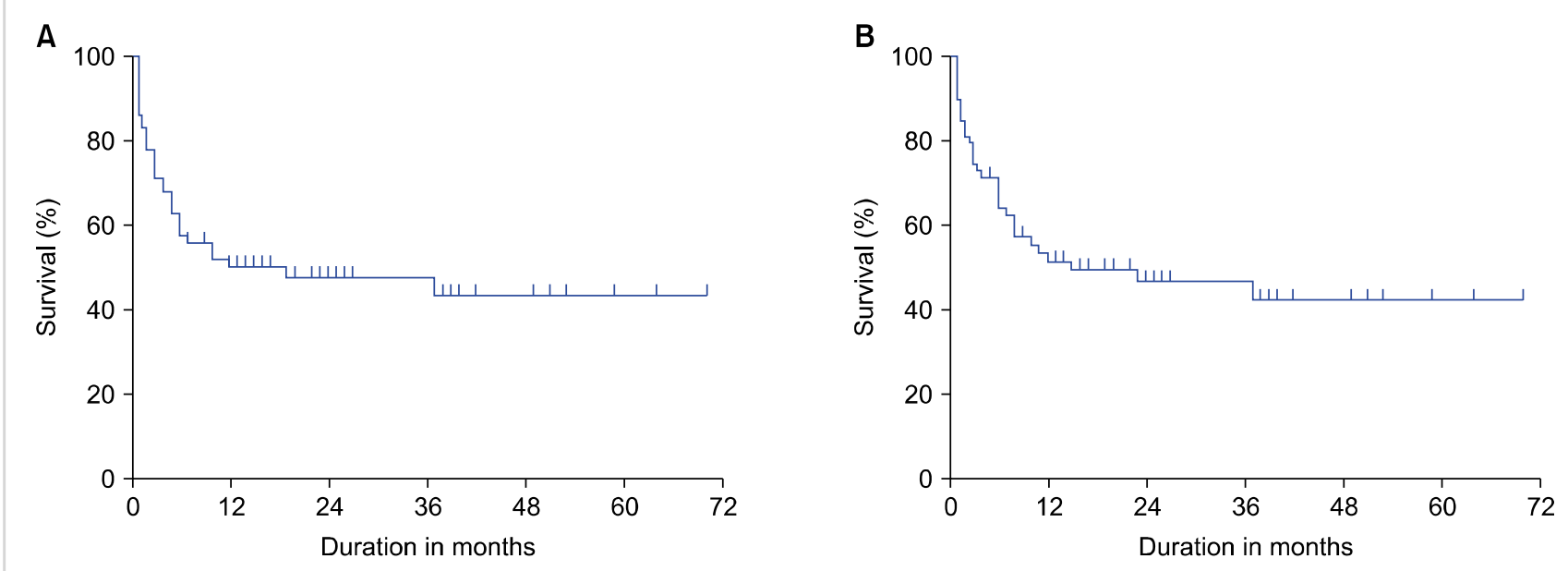

Fig. 2. Kaplan-Meier survival curves for the entire cohort for (A), progression-free survival and (B) overall survival. 

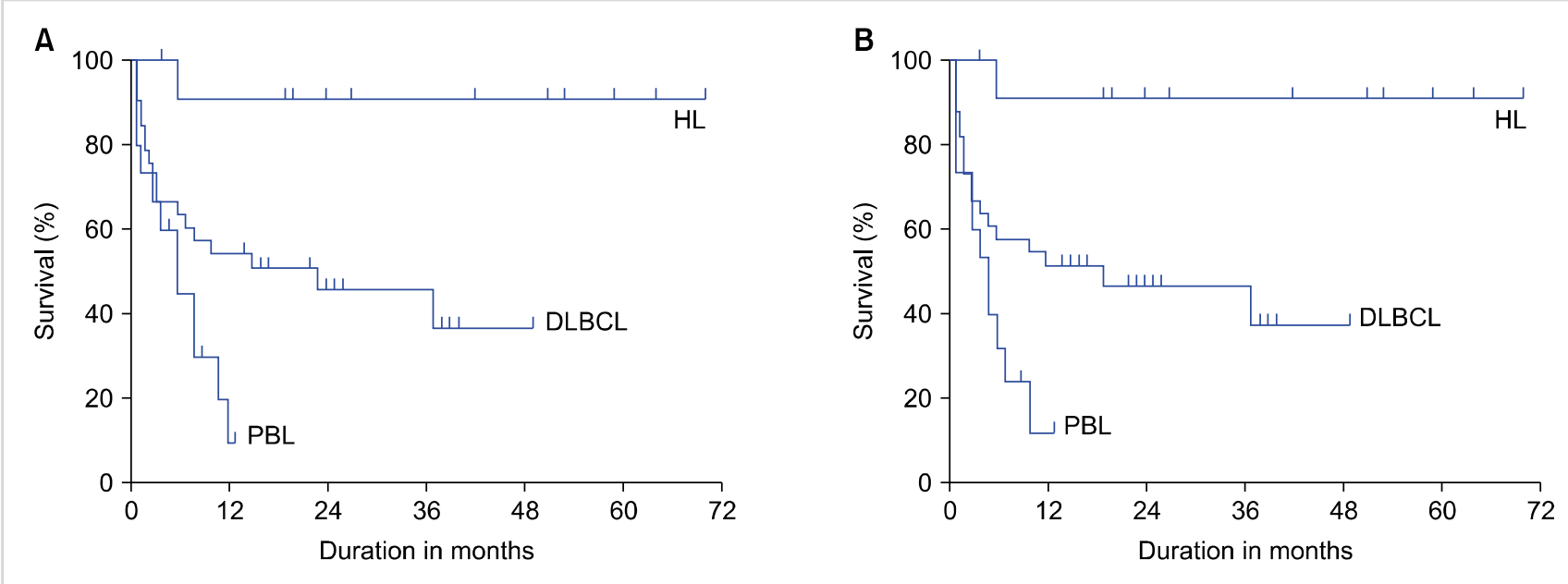

Fig. 3. Histology-specific Kaplan-Meier survival curves for (A), progression-free survival and (B) overall survival.

\section{DISCUSSION}

Cancer was related to HIV infection even before the identification of HIV itself; in fact, patients with Kaposi's sarcoma were among the first, which led to the discovery of HIV [7]. PLHA are at higher risks of a variety of cancers compared to those in their age and sex-matched counterparts in the general population. HIV-AIDS related cancers are divided into AIDS-defining and non-AIDS defining by the Centers for Disease Prevention and Control (CDC) [8]. Kaposi's sarcoma, certain types of NHL, and invasive cervical cancer are labeled as AIDS-defining cancers by the CDC. There is no clarification about which particular subtypes should be included in AIDS-defining NHL but high-grade lymphomas are conventionally considered ADL while HL and most low-grade lymphomas are considered NADL. In the current study, high-grade B-cell lymphomas and certain NHL which are particularly associated with HIV/AIDS such as PCNSL and PEL were considered ADL, while HL and FL were considered NADL. PTCL, even though a high-grade NHL, was included as a NADL because of the lack of standard definitions and our series had only one case of PTCL NOS.

The spectrum of HIV/AIDS-related malignancies in India differs from that reported in the Western literature [5, 9, 10]. This difference may be due to the low prevalence of human herpesvirus 8 in the Indian population or to differential patterns of HIV transmission, with the heterosexual route the most common in India [11]. Survival of PLHA is increasing with combination ART and is now comparable to that of the general population. India is a pioneer in affordable generic ART; as per the 2018 UNAIDS report, 56\% of PLHA are receiving ART, with a goal of reaching $90 \%$ [1]. The natural history of HIV is changing in India with the use of ART and improved prophylaxis and treatment of opportunistic infections. With improved life expectancy, the burden of cancer in PLHA will continue to increase in upcoming years. There is a paucity of data on the epidemi- ology and management of AIDS-related malignancies in India. The median age at presentation in our study was 40.5 years for the entire cohort, which is a decade earlier than that in the HIV-negative population [12]. The male to female ratio of 2:1 in our study may represent the pattern of HIV prevalence in the Indian general population, which shows M: F ratio of $1.36: 1$ or there might be an actual underlying biological difference depending on gender.

PLHA were excluded in most clinical trials evaluating the role of various chemotherapy regimens for lymphomas. There are several controversies regarding the treatment of ARL, including the optimal regimen of choice, dose intensity, use of rituximab, the role of CNS prophylaxis, and continuation of ART during lymphoma treatment. There are no clear guidelines for the treatment of HIV-associated malignancies from any national or international groups, except for the British HIV Association guidelines in 2014, with many unanswered questions [13]. Treatment decisions are largely based on retrospective series and a few prospective phase $2 / 3$ studies. Initial reports suggested that the increased mortality in ARL patients treated with standard dose chemotherapy was due to increased infectious complications resulting from chemotherapy-induced immune suppression combined with low baseline immune function [14]. Based on a study from the AIDS Malignancy Consortium (AMC), the reduced dose treatment offered significant less hematological toxicity with similar efficacy in ARL. This trial used mBACOD (methotrexate, bleomycin, adriamycin, cyclophosphamide, vincristine, and dexamethasone) regimen and subsequent studies in HIV-negative patients showed CHOP to be equally effective and less toxic than mBACOD [15]. CHOP became the standard of care for high-grade lymphomas before the introduction of rituximab in HIV-negative patients. Various reports suggest that while CHOP can be used in ARL, the outcomes are less optimal compared to those in HIV-negative patients [16]. Many of these early studies did not include granulocyte-colony stimulating factor (G-CSF) support or CNS and Pneumocystis jiroveci pneumo- 
nia prophylaxis, which might have affected outcomes. Studies testing infusional regimens with DA-EPOCH and CDE (cyclophosphamide, doxorubicin, and etoposide) were tried in a limited number of patients to improve the poor results in ARL and were reported to improve outcomes [17, 18]. There are no phase 3 studies comparing various regimens to guide the choice of an optimal chemotherapy backbone. In our study, two-thirds of DLBCL and PBL patients received CHOP, while DA-EPOCH was used in 11 (22.9\%) patients. All patients received prophylaxis against $P$. jiroveci pneumonia and oral antibiotics during chemotherapy. All DA-EPOCH patients received prophylactic G-CSF for seven days, while G-CSF support was individualized for patients on CHOP. Hematological and non-hematological toxicities, treatment-related mortality, and use of supportive care were significantly higher in DA-EPOCH than those in CHOP, without improvement in clinical outcomes.

Rituximab is an integral part of the management of immunocompetent patients with CD-20-positive NHL; however, the evidence is conflicting for patients with ARL. The AMC 010 trial, the only phase 3 study comparing CHOP to R-CHOP, did not show improvements in the clinical outcomes of ARL patients receiving rituximab [19]. There was a significant increase in treatment-related infectious deaths in patients receiving rituximab. The authors concluded that even though rituximab improved response rates, the increased mortality offset the benefits. One particular drawback of this study was the use of maintenance rituximab, which is not standard practice for high-grade B-cell NHL. Subsequent analysis showed that when patients with severe immune-deficiency (i.e. CD4 count $<50$ cells $/ \mu \mathrm{L}$ ) were excluded, infectious deaths did not differ significantly between the two groups. In a pooled analysis of individual patient data from 19 prospective trials, rituximab use was associated with a significant increase in CR rates and improved PFS and OS in patients with CD4 count $\geq 50$ cells/ $\mu \mathrm{L}$ but not in those with a count $<50$ cells $/ \mu \mathrm{L}$, in both univariate and multivariate analysis [20].

Our center is one of the regional cancer care centers providing comprehensive tertiary level care for cancer patients. Most of the patient population are of low socio-economic status and receive treatment under a variety of schemes funded by central or state governments. At the time of this writing, these schemes do not cover many of the high-cost cancer medicines including monoclonal antibodies like rituximab. In India, around $75 \%$ of the health care expenditure is borne by patients and their households as out-of-pocket expenditures, which may be direct medical, non-medical, or indirect costs [21]. ARL patients have to bear the brunt of two chronic debilitating diseases simultaneously, which further increases their socio-economic stress. This might have important implications on clinical outcomes including OS for these patients. In our study, only six of the 33 DLBCL patients $(18.18 \%)$ received a rituximab-based chemotherapy regimen because of economic constraints. Continuation of treatment after failure of first-line therapy was dismal, with only four patients receiving subsequent lines of treatment for their lymphoma. The absolute difference between the median PFS and OS was only four months for DLBCL and one month for PBL because the majority of patients who progressed on first-line treatment defaulted for further treatment and data for OS were censored at the time of the last follow up visit. This poor adherence to subsequent treatment due to various socio-economic constraints and significant loss to follow-up is reflected in the nearly identical Kaplan-Meier graphs of PFS and OS, which is a major limitation of our study. Despite these shortcomings, the median OS for DLBCL was 23 months, similar to that for the HIV-negative population in our previous series [12]. The observed treatment-related mortality in the present study of $17.2 \%$ was high. The majority of induction deaths had advanced stage disease, poor PS, high IPI, and low CD4 count at the start of treatment.

ART has changed the landscape of HIV infection, making it "just another chronic illness". Concerns were raised regarding the concomitant use of ART with chemotherapy in patients with ARL because of the possible increased toxicity, drug metabolism, and drug interactions. Unfortunately, literature is scarce on this topic. Most studies showed no clinically adverse effects on drug metabolism for concurrent ART and conventional chemotherapy and suggested that most ART regimens can be safely implemented in PLHA undergoing treatment for cancer, with the exception of zidovudine [22, 23]. Similarly, drug interactions are not of major clinical concern with ART and chemotherapy use except for protease inhibitors and cobicistat. Immune dysregulation because of HIV infection is basic pathobiology behind the development of lymphoma in PLHA. ART improves immune function by suppressing viral replication; by doing, so it curtails the risk of severe immune suppression and subsequent development of lymphoma. ART use in ARL patients decreases the risk of developing opportunistic infections during treatment, thus improving the tolerability of chemotherapy. A study evaluating temporal trends in ARL presentation and survival outcomes in a large cohort of PLHA reported important changes in demographic, virologic, and histologic patterns over a 15-year period [24]. The study concluded that survival outcomes have not significantly changed in the modern ART era. At the same time, multiple reports have shown ART to be associated with significant improvement in clinical outcomes in ARL including response rates and OS [19, 25-27]. There might also be biological differences in the lymphoma developing in patients on ART compared to that in treatment-naïve PLHA. In our study, 40 patients were already on ART at the time of ARL diagnosis while in 30 patients, lymphoma was the presentation of the underlying HIV. All patients received fixed-drug combination ART under the NACO as per national guidelines and continued it with chemotherapy. We could not analyze data for different ART regimens and their relationship with drug metabolism and drug interactions due to infrastructure limitations.

To our knowledge, this is the largest retrospective analysis of ARL from Indian literature. ARL is a common malignancy in PLHA in India. With the increasing reach of the National 
Aids Control Programme and ART coverage across the country along with the improved management of opportunistic infections, ARL will be a major cause of morbidity and mortality in upcoming years. ADL had a significantly lower CD4 count than that of NADL. ARL is a heterogeneous disease with histologic subtype a major determinant of clinical outcome. With standard treatment protocols and proper supportive care, these patients can have similar clinical outcomes to those of their HIV-negative counterparts with lymphoma. With a lack of standard national or international guidelines, there is an unmet and urgent need for prospective multicenter collaborative trials which might shed light on the management of this often neglected and under-represented patient population.

\section{ACKNOWLEDGMENTS}

Dr. Snehal Chavhan. Assistant Professor, Department of Community Medicine, Dr. D. Y. Patil Medical College, Pimpri, Maharashtra, India for help with statistical analysis of data.

\section{Authors' Disclosures of Potential Conflicts of Interest}

No potential conflicts of interest relevant to this article were reported.

\section{REFERENCES}

1. UNAIDS. UNAIDS Data 2018. Geneva, Switzerland: UNAIDS, 2018. (Accessed February 20, 2019, at http://www.unaids.org/ sites/default/files/media_asset/unaids-data-2018_en.pdf).

2. National AIDS Control Organization. Annual Report 2016-17. New Delhi, India: Government of India, 2018. (Accessed February 20, 2019, at http://naco.gov.in/sites/default/files/NACO\%20A NNUAL\%20REPORT\%202016-17.pdf).

3. Central Drugs Standard Control Organization. National Health Policy. New Delhi, India: Government of India, 2017. (Accessed February 20, 2019, at https://mohfw.gov.in/sites/default/files/ 9147562941489753121.pdf).

4. Crum-Cianflone N, Hullsiek KH, Marconi V, et al. Trends in the incidence of cancers among HIV-infected persons and the impact of antiretroviral therapy: a 20-year cohort study. AIDS 2009; 23:41-50.

5. Paul TR, Uppin MS, Uppin SG, et al. Spectrum of malignancies in human immunodeficiency virus - positive patients at a Tertiary Care Centre in South India. Indian J Cancer 2014;51:459-63.

6. Prem S, Narayanan G, Puthuveettil J, Jayasree K, Vijayalakshmi K. Spectrum of HIV/AIDS-associated cancers in South India. J Clin Oncol (ASCO Annual Meeting Abstracts) 2014;32(Suppl 15): e12534.

7. Centers for Disease Control (CDC). A cluster of Kapos's sarcoma and Pneumocystis carinii pneumonia among homosexual male residents of Los Angeles and Orange Counties, California.
MMWR Morb Mortal Wkly Rep 1982;31:305-7.

8. 1993 revised classification system for HIV infection and expanded surveillance case definition for AIDS among adolescents and adults. MMWR Recomm Rep 1992;41:1-19.

9. Dhir AA, Sawant S, Dikshit RP, et al. Spectrum of HIV/AIDS related cancers in India. Cancer Causes Control 2008;19:147-53.

10. Haleshappa RA, Koppaka D, Lakshmaiah KC, et al. Patterns of malignancies in patients with HIV-AIDS: a single institution observational study. J Community Support Oncol 2018;16: e188-92.

11. Biggar RJ, Chaturvedi AK, Bhatia K, Mbulaiteye SM. Cancer risk in persons with HIV/AIDS in India: a review and future directions for research. Infect Agent Cancer 2009;4:4.

12. Babu SM, Garg S, Kanakasetty GB, Kuntegowdanahalli LC, Dasappa L, Rao SA. Diffuse large B-cell lymphoma: A retrospective study from a regional care center in South India. Indian J Cancer 2018;55:66-9.

13. Bower M, Palfreeman A, Alfa-Wali M, et al. British HIV Association guidelines for HIV-associated malignancies 2014. HIV Med 2014;15(Suppl 2):1-92.

14. Kaplan LD, Abrams DI, Feigal E, et al. AIDS-associated non-Hodgkin's lymphoma in San Francisco. JAMA 1989;261: 719-24.

15. Kaplan LD, Straus DJ, Testa MA, et al. Low-dose compared with standard-dose m-BACOD chemotherapy for non-Hodgkin's lymphoma associated with human immunodeficiency virus infection. National Institute of Allergy and Infectious Diseases AIDS Clinical Trials Group. N Engl J Med 1997;336:1641-8.

16. DeMario MD, Liebowitz DN. Lymphomas in the immunocompromised patient. Semin Oncol 1998;25:492-502.

17. Little RF, Pittaluga S, Grant N, et al. Highly effective treatment of acquired immunodeficiency syndrome-related lymphoma with dose-adjusted EPOCH: impact of antiretroviral therapy suspension and tumor biology. Blood 2003;101:4653-9.

18. Sparano JA, Lee S, Chen MG, et al. Phase II trial of infusional cyclophosphamide, doxorubicin, and etoposide in patients with HIV-associated non-Hodgkin's lymphoma: an Eastern Cooperative Oncology Group Trial (E1494). J Clin Oncol 2004;22:1491-500.

19. Kaplan LD, Lee JY, Ambinder RF, et al. Rituximab does not improve clinical outcome in a randomized phase 3 trial of $\mathrm{CHOP}$ with or without rituximab in patients with HIV-associated non-Hodgkin lymphoma: AIDS-Malignancies Consortium Trial 010. Blood 2005;106:1538-43.

20. Barta SK, Xue X, Wang D, et al. Treatment factors affecting outcomes in HIV-associated non-Hodgkin lymphomas: a pooled analysis of 1546 patients. Blood 2013;122:3251-62.

21. Mohanti BK, Mukhopadhyay A, Das S, Sharma K, Dash S. Estimating the economic burden of cancer at a tertiary public hospital: a study at the All India Institute of Medical Sciences. New Delhi, India: Indian Statistical Institute, Delhi Centre, 2011. (Accessed February 20, 2019, at https://www.isid.ac.in/ abhiroop/res_papers/WORKING_PAPERS_files/cancer.pdf).

22. Tan B, Ratner L. The use of new antiretroviral therapy in combination with chemotherapy. Curr Opin Oncol 1997;9: 455-64.

23. Torres HA, Mulanovich V. Management of HIV infection in patients with cancer receiving chemotherapy. Clin Infect Dis 
2014;59:106-14.

24. Gopal S, Patel MR, Yanik EL, et al. Temporal trends in presentation and survival for HIV-associated lymphoma in the antiretroviral therapy era. J Natl Cancer Inst 2013;105:1221-9.

25. Weiss R, Mitrou P, Arasteh K, et al. Acquired immunodeficiency syndrome-related lymphoma: simultaneous treatment with combined cyclophosphamide, doxorubicin, vincristine, and prednisone chemotherapy and highly active antiretroviral therapy is safe and improves survival-results of the German
Multicenter Trial. Cancer 2006;106:1560-8.

26. Lim ST, Karim R, Tulpule A, Nathwani BN, Levine AM. Prognostic factors in HIV-related diffuse large-cell lymphoma: before versus after highly active antiretroviral therapy. J Clin Oncol 2005; 23:8477-82.

27. Thirlwell C, Sarker D, Stebbing J, Bower M. Acquired immunodeficiency syndrome-related lymphoma in the era of highly active antiretroviral therapy. Clin Lymphoma 2003;4: 86-92. 